$\frac{46}{410} .95 \mathrm{~g} 5(2)$

PREPARED FOR THE U.S. DEPARTMENT OF ENERGY, UNDER CONTRACT DE-AC02-76-CHO-3073

PPPL-3052

- PPPL-3052 UC-427

\title{
A GENERALIZED GYROKINETIC POISSON SOLVER
}

\author{
BY
}

Z. LIN AND W.W. LEE

MARCH 1995

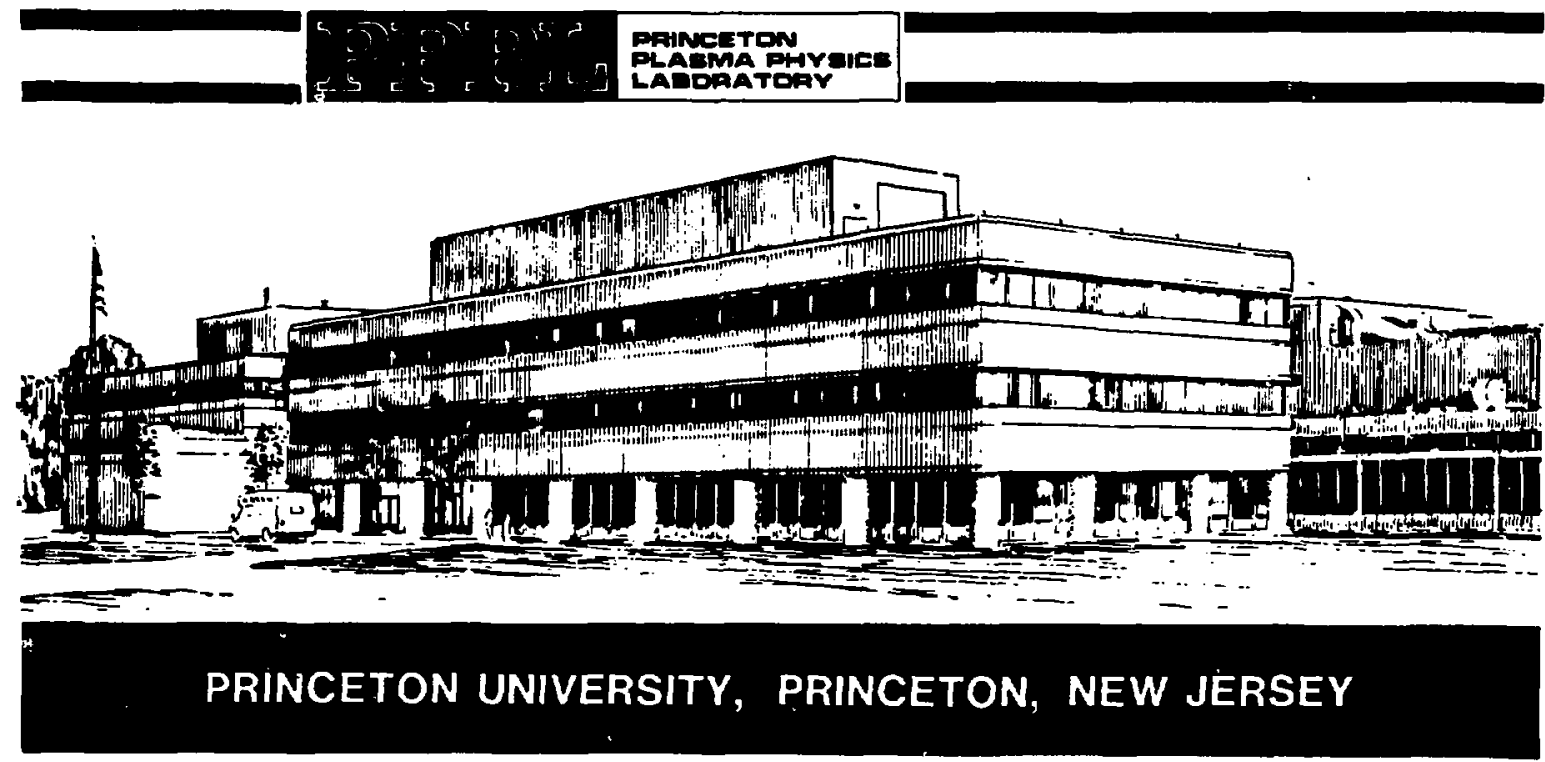




\section{NOTICE}

This report was prepared as an account of work sponsored by an agency of the United States Government. Neither the United States Government nor any agency thereof, nor any of their employees, makes any warranty, express or imiplied, or assumes any legal liability or responsibility for the accuracy, completeness, or usefulness of any information, apparatus, product, or process disclosed, or represents that its use would not iniringe privately owned rights. Reference herein to any specific commercial produce, process, or service by trade name, trademark, manufacturer or otherwise, does not necessarily constitute or imply its endorsement, recommendatic , or favoring by the United States Government or any agency thereof. The views anci opinions of authors expressed herein do not necessarily state or reflect those of the United States Government or any agency thereof.

\section{NOTICE}

This report has been reproduced from the best available copy. Available in paper copy and microfiche.

Number of pages in this report: 24

DOE and DOE contractors can obtain copies of this report from:

Office of Scientific and Technical Information

P.O. Box 62

Oak Ridge, TN 37831;

(615) 576-8401.

This report is publicly available from the:

National Technical Information Service

Department of Commerce

5285 Port Royal Road

Springfield, Virginia 22161

(703) $487-4650$ 


\title{
A Generalized Gyrokinetic Poisson Solver
}

\author{
Z. Lin and W. W. Lee \\ Princeton Plasma Physics Laboratory \\ Princeton University \\ P.O. Box 451 \\ Princeton, NJ 08543-0451
}

\begin{abstract}
A generalized gyrokinetic Poisson solver has been developed, which employs local operations in the configuration space to compute the polarization density response. The new technique is based on the actual physical process of gyrophase-averaging. It is useful for nonlocal simulations using general geometry equilibrium. Since it utilizes local operations rather than the global ones such as FFT, the new method is most amenable to massively parallel algorithms.
\end{abstract}

\section{DISCLAIMER}

\begin{abstract}
This report was prepared as an account of work sponsored by an agency of the United States Government. Neither the United States Government nor any agency thereol, nor any of their employees, makes any warranty, express or implied, or 2ssumes any legal liability or responsibility for the accuracy, completeness, or usefulness of any information, apparatus, product, of process disclosed, or represents that its use would not infringe privately owned rights. Reference herein to any specific commercial product, process, or service by trade name, trademark, manufacturer, or otherwise does not necessarily constitute or imply its endorsement, recom. mendation, or favoring by the United States Government or any agency thereof. The views and opinions of nuthors expressed herein do not necesserily state or reflect thoce of the Unitod States Government or any agency thereof.
\end{abstract}

\section{MASTER}

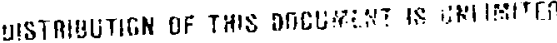




\section{INTRODUCTION}

In this paper, a generalized gyrokinetic Poisson solver suitable for arbitrary geometry is presented. This is an extension of the commonly used spectral methods for solving the gyrokinetic Poisson equation. Since the original equation is an integral equation in the configuration space, the spectral results, strictly speaking, are only valid as local solutions. The new technique is based on the actual physical process of gyrophase-averaging and employs local operations in the configuration space to compute the polarization density response in the equation. Therefore, it can take into account the spatial dependence in the equation and is useful for nonlocal simulations as well as those associated with general geometry equilibrium. It is also most suitable for massively parallel computing, since the new scheme favors local operations by minimizing global communications.

For simplicity, let us study the gyrokinetic Vlasov-Poisson system in slab geometry which takes the form (neglect nonlinear polarization term and terms of order of $\left.\vartheta\left(k_{\perp} \rho\right)^{4}\right)$ of

$$
\frac{\partial F}{\partial t}+v_{\|} \hat{b} \cdot \frac{\partial F}{\partial \mathbf{R}}-\frac{q}{m \Omega} \frac{\partial \bar{\Phi}}{\partial \mathbf{R}} \times \hat{b} \cdot \frac{\partial F}{\partial \mathbf{R}}-\frac{q}{m} \frac{\partial \bar{\Phi}}{\partial \overline{\mathbf{R}}} \cdot \hat{b} \frac{\partial F}{\partial v_{\|}}=0,
$$

and

$$
\frac{\tau}{\lambda_{D}^{2}}(\Phi-\tilde{\Phi})=4 \pi e\left(\bar{n}_{i}-n_{e}\right) .
$$

Here, $F\left(\mathbf{R}, \mu, v_{\|}, t\right)$ is the gyrocenter distribution function, which is independent of gyrophase, $\mathbf{R} \equiv \mathbf{x}-\vec{\rho}, \vec{\rho} \equiv-v_{\perp} \times \hat{\mathbf{b}} / \Omega, \Omega \equiv q B / m c, \hat{\mathbf{b}} \equiv \mathbf{B} / B$, $\mathrm{B}$ is the external magnetic field, $\mu \equiv v_{\perp}^{2} / 2, \mathbf{v}_{\| \mid}=v_{\| \mid} \hat{\mathrm{b}}, q$ is the signed charge, $\lambda_{D} \equiv \sqrt{T_{e} / 4 \pi n_{0} e^{2}}$ is the Debye length, $\tau \equiv T_{e} / T_{i}$, subscripts $e$ and $i$ denote species, $\Phi(\mathbf{x}, t)$ is the electrostatic potential, $\bar{\Phi}$ represents gyrophase averaged potential, and $\tilde{\Phi}$ is defined as the second gyrophase-averaged povential. Specifically,

$$
\overline{\boldsymbol{\Phi}}(\mathbf{R})=\frac{1}{2 \pi} \int \Phi(\mathbf{x}) \delta(\mathbf{x}-\mathbf{R}-\rho) d \mathbf{x} d \alpha,
$$

where $\alpha$ is the gyrophase angle and $\mathbf{R}$ is held fixed in the integration, and

$$
\tilde{\Phi}(\mathbf{x})=\frac{1}{2 \pi} \int \overline{\mathbf{\Phi}}(\mathbf{R}) F_{M i}\left(\boldsymbol{e R}, \mu, v_{\|}\right) \delta(\mathbf{R}-\mathbf{x}+\rho) d \mathbf{R} d \mu^{\prime} d v_{\|} d \alpha
$$

with $\mathrm{x}$ now held fixed in the integration. In Eq. 4, $F_{M i}$ is assumed to be Maxwellian in $v_{\perp}$ and is spatially slow varying, i.e., $\epsilon \ll 1$. The gyrophase 
averaged ion number density is defined as

$$
\overline{\mathbf{n}}_{i}(\mathbf{x})=\frac{1}{2 \pi n_{0}} \int F_{i}(\mathbf{R}) \delta(\mathbf{R}-\mathbf{x}+\rho) d \mathbf{R} d \mu d v_{\|} d \alpha
$$

and $n_{e}$ is the electron number density based on $F_{e}$ in the limit of $\rho \rightarrow 0$. The background number density $n_{0}$ comes from $F_{M i}$ assuming $e x \approx \epsilon \mathbf{R}$.

In this system, the gyrophase-averaged distribution function is a function of gyrocenter variables; e.g. $F=F\left(\mathbf{R}, \mu, v_{\|}, t\right)$, while the electrostatic potential is defined in particle or laboratory coordinates, $\Phi=\Phi(x, t)$. Thus, to solve the gyrokinetic Vlasov-Poisson system, one has to develop numerical algorithm to expedite the transformation. In the Fourier space, the coordinate transformation can simply be carried out by using

$$
\Phi(\boldsymbol{x})=\sum_{k} \boldsymbol{\Phi}_{k} e^{i k \cdot x}
$$

and applying it to Eq. 3 to obtain

$$
\bar{\Phi}(\mathbf{R})=\sum_{k} \Phi_{k} J_{0}\left(\frac{k_{\perp} v_{\perp}}{\Omega}\right) e^{i \mathbf{k} \cdot \mathbf{R}},
$$

where $J_{0}$ is the ordinary Bessel function, and

$$
\int_{0}^{2 \pi} \exp ( \pm i k \cdot \rho) d \alpha / 2 \pi=J_{0}\left(k_{\perp} v_{\perp} / \Omega\right)
$$

is utilized in this transformation. Likewise, $\tilde{\Phi}$ in Eq. 4 can be calculated in the Fourier space by assuming a spatially independent Maxwellian equilibrium and it becomes

$$
\tilde{\Phi}(\mathbf{x})=\sum_{k} \Phi_{k} \Gamma_{0}(b) e^{i k \cdot x},
$$

where $\Gamma_{0}(b)=I_{0}(b) e^{-b}, b \equiv\left(k_{\perp} \rho_{i}\right)^{2}, \rho_{i} \equiv \sqrt{T_{i} / m_{i}} / \Omega_{i}$, and $I_{0}$ is the modified Bessel function. The term $\Gamma_{0}$ comes from

$$
\Gamma_{0}(b)=\int J_{0}^{2}\left(\frac{k_{\perp} v_{\perp}}{\Omega}\right) F_{M i}(\mu) d \mu,
$$

which is the result of two gyrophase averaging processes with respect to a Maxwellian background. Since the Debye shielding term in the gyrokinetic Poisson equation is usually neglected in the gyrokinetic particle simulations of low-frequency physics, the resulting equation is an integral equation and, 
for simple geometry, it can be transformed in Fourier k-space and solved by the FFT method.

However, the use of Fourier transform is not always practical, because it involves the convolution between the configuration space and the velocity space through the $J_{0}$ term. For example, a better and more practical way is to carry out the transformation of Eq. 3 in the configuration space by an averaging process for $\Phi(\mathbf{x})$ assuming that each particle is represented by a uniformly charged ring with a radius of $\rho=v_{\perp} / \Omega$. Thus, integrating along the ring with sufficient accuracy, one recovers Eq. 3. As shown in Ref. 1 , it is usually sufficient to use four points along the ring to achieve the desired accuracy for $k_{\perp} \rho_{i} \leq 2$. As for solving Eq. 2, because the term involving $\Gamma_{0}$ in Eq. 7 can be expressed as analytic functions in the $k$-space, the spectral approach is more practical. However, this approach is not applicable in general geometry, i.e., realistic tokamak equilibrium. Even in the simple case of circular cross section toroidal geometry, the FFT approach has difficulties in implementing appropriate boundary condition and evaluating the mode spectrum. Furthermore, the k-space gyrokinetic Poisson equation is derived based on the two-spatial-scale assumption and one neglects the spatial dependence of the equilibrium profile. In reality $\Gamma_{0}$ is a function of spatial coordinates when a perpendicular temperature gradient exists. Therefore, it is of interest in a realistic simulation to include the variation of temperature across the global simulation box. Various schemes have thus been developed to address these two problems. LeBrun et al. ${ }^{2}$ employed a Padé approximation for the $\Gamma_{0}$ in Eq. 7 and solve the resulting gyrokinetic Poisson equation as a second order differential equation. $\mathrm{Li}$ et $a l^{3}$ used the spectral approach in two directions, i.e., poloidal and toroidal $(\theta, \phi)$, and made the Bessel function expansion in the minor radius direction for plasmas with a circular cross section. However, these approaches are not satisfactory with respect to the proper treatment of non-local effect and the extension to general equilibrium geometry.

In this paper, we present a new method which enables us to carry out the transformation of $\tilde{\Phi}$ in the configuration space in the same spirit as the calculation of $\bar{\Phi}$. We adapt a local operation to calculate $\bar{\Phi}$ using the 4-point averaging procedure in configuration space. Good agreement with the FFT method is obtained in a simple test case of $\eta_{i}$ instability nonlinear simulation using this new technique in a shearless slab where the FFT method is valid. In toroidal geometry, the electrostatic potential resulting from a given charge density is calculated both analytically and numerically. The results 
using the new solver is much closer to the exact analytic solution than that of the FFT method when the same number of grid points is used. The importance of profile variation is also demonstrated in this calculation. The computational cost depends on the complexity of the geometry. The timing of the new solver is better than that of the FFT method in a shearless slab with adiabatic electrons, and is comparable to that of particle pushing in a realistic tokamak equilibrium where the FFT method is not valid.

The remainder of this paper is organized as follows. Section II describe the non-FFT approach to gyrokinetic Poisson equation. Numerical algorithm and benchmark results for both slab and cylindrical geometries are presented in Section III. Section IV summarizes the main findings of this paper.

\section{GENERALIZED GYROKINETIC POISSON SOLVER}

The key idea of the present approach is to calculate $\bar{\Phi}\left(\mathbf{x}_{g}\right)$ from a given $\Phi\left(x_{g}\right)$, where $x_{g}$ represents the coordinates of the grid points in the simulation, and cast Eq. 2 in a matrix form to be solved by a iteration scheme or a direct matrix inversion method. In this way, the gyrokinetic Poisson equation is solved in the configuration space directly without transforming to the k-space.

Our starting point of analysis is Eq. 4, which relates $\tilde{\mathbf{\Phi}}(\mathbf{x})$ on any point in configuration space to the guiding center potential $\bar{\Phi}(\mathbf{R})$ through a uniformly distributed background $F_{M i}$. We can replace the Maxwellian distribution function by a sum of infinite series of delta functions which are equally spaced in the perpendicular velocity space $\left(v_{\perp}\right)$ and weighted by an envelop of normalizeci Gaussian function. To calculate $\tilde{\Phi}\left(x_{g}\right)$ on the grid point $x_{g}$, we interpret each delta function inside the integration of Eq. 4 as a uniform ring with a radius of $r=p_{i} v_{\perp} / v_{t h i}$ and centered at $x_{g}$ (solid circle in Fig. 1). Then $\bar{\Phi}\left(x_{g}\right)$ is simply the average of the gyro-averaged guiding center potential $\overline{\boldsymbol{\Phi}}\left(\mathbf{R} \equiv \mathbf{x}_{g}-\vec{\rho}\right)$ on this ring. On the other hand, $\overline{\boldsymbol{\Phi}}\left(\mathbf{x}_{g}-\vec{\rho}\right)$ of each point on this ring is first evaluated by averaging the potential $\Phi(\mathbf{x})$ over another uniform ring centered at this point with the same radius (dashed circle in Fig. 1). These averaging processes can be done by the 4-point average procedure which is exact up to $k_{\perp} \rho_{i}=2$.

With regard to the physical interpretation of this approach, let us examine how we arrive at Eq. 4. Originally, $\Phi(\mathbf{x})-\bar{\Phi}(\mathbf{x})$ is defined as the difference between the potentials perceived by the particle and the guiding 
center. ${ }^{4}$ For a known $\Phi(\mathbf{x})$, we can calculate $\overline{\boldsymbol{\Phi}}(\mathbf{R})$ for each guiding center by averaging the $\Phi(x)$ on its uniformly charged ring. This averaged potential is then deposited back onto this ring, and it represents this particle's contribution to $\tilde{\Phi}(\mathbf{x})$ on every point of this ring. Summing up the contribution from all particles, we obtained $\tilde{\Phi}(\mathbf{x})$. To facilitate the computation, we first note that we only need to calculate $\tilde{\Phi}(x)$ at each grid point $x_{g}$ instead of every point in the configuration space, then we re-write the original gyrokinetic Poisson equation to the form as in Eq. 4 with a simplification in which the background distribution function is replaced by a spatially uniform Maxwellian in $v_{\perp}$. The summation process now involves an infinite number of particles with the Maxwellian distribution at every point in the configuration space. However, not all particles can contribute to $\tilde{\Phi}\left(\mathbf{x}_{g}\right)$ at a grid point. Those particles with a particular gyroradius $\rho_{0}$ that can contribute to $\tilde{\Phi}\left(\mathbf{x}_{g}\right)$ only come from a uniform ring which centers at $\mathbf{x}_{g}$ with a radius $\rho_{0}$. As a result, we can change the sequence of the summation process. Rather than summing over each particle contribution to $\tilde{\Phi}(\mathbf{x})$ on its uniform charge ring, we can focus on $\tilde{\Phi}\left(\mathbf{x}_{g}\right)$ at one grid point, first sum over all particles with a particular gyroradius that can make contribution to $\tilde{\Phi}\left(\mathbf{x}_{g}\right)$, and then add up the contribution from all possible gyroradius. Thus we recover the procedure outlined in the last paragraph.

It is computationally prohibited to sample the whole Maxwellian distribution function by an infinite number of delta functions. Fortunately, we need only a few delta functions to mimic the Maxwellian distribution function since it decays exponentially for higher velocities. To verify this claim, we replace the Maxwellian by a sum of finite series of delta functions in Eq. 7, i.e.,

$$
\Gamma_{0}\left(k_{\perp}^{2} \rho_{i}^{2}\right) \simeq \int J_{0}^{2}\left(\frac{k_{\perp} v_{\perp}}{\Omega}\right) \sum_{j} c_{j} \delta\left(\mu-\mu_{j}\right) d \mu=\sum_{j} c_{j} J_{0}^{2}\left(\frac{v_{\perp j}}{v_{\ell h i}} k_{\perp} \rho_{i}\right)
$$

where $c_{j}$ represents the Gaussian envelope and $v_{\perp j}$ is the perpendicular velocity of the representative ring and $v_{t h i} \equiv \sqrt{T_{i} / m_{i}}$ is the ion thermal velocity. This set of $c_{j}$ 's and $v_{\perp j}$ 's is approaching the exact Maxwellian distribution function when infinite number of delta functions is used. Practically, Eq. 8 is realized by choosing a finite number of $c_{j}$ 's and $v_{\perp i}$ 's such that the following function is minimized,

$$
\epsilon\left(c_{j}, v_{\perp j}\right)=\int_{0}^{x_{\max }}\left[\Gamma_{0}\left(x^{2}\right)-\sum_{j} c_{j} J_{0}^{2}\left(x v_{\perp j} / v_{t h i}\right)\right]^{2} d x
$$


where $x \equiv k_{\perp} \rho_{i}$ and $x_{m a x}$ corresponds to the maximum wave number of interest. To reduce the computational cost, it is, therefore, desirable to fit Eq. 8 for arbitrary $k_{\perp}$ 's by as few rings as possible. Fortunately, $k_{\perp}$ only varies in the range of $0<k_{\perp} \rho_{i}<\pi \rho_{i} / \Delta x$, where $\Delta x$ is the grid spacing, and the mode amplitude is usually small for the $k_{\perp} \rho_{i}>1$ modes. Thus, only a few ringe are needed in Eq. 8. Practically, three rings work quite well over the range of $0<k_{\perp} \rho_{i}<2$. In Fig, 2, the approximate $\Gamma_{0}$ (dashed line), using $c=(0.486,0.248,0.266)$ and $v_{\perp} / v_{t h i}=(\sqrt{2} / 2, \sqrt{2}, 3 \sqrt{2} / 2)$, is compared to the exact $\Gamma_{0}$ (solid line).

The number of free parameters of $c_{j}$ 's and $v_{\perp j}$ 's can be reduced by 2 when we minimize the $\epsilon$ to be zero in the long wave length limit. We match the first two terms for the small $x$ expansion for the Bessel function, i.e., $J_{0}\left(x v_{\perp j} / v_{t h i}\right) \sim 1-\left(x v_{\perp j} / v_{t h i}\right)^{2} / 4+\cdots$, and the Padé approximation of $\Gamma_{0}\left(x^{2}\right) \sim 1 /\left(1+x^{2}\right) \sim 1-x^{2}+\cdots$, and arrive at the following constraints,

$$
\sum_{j} c_{j}=1
$$

and

$$
\sum_{j} \frac{1}{2} c_{j}\left(v_{\perp}^{j} / v_{t h i}\right)^{2}=1
$$

These constraint conditions are most useful for long wavelength modes.

\section{NUMERICAL IMPLEMENTATION AND TEST CASE RESULTS}

\section{A. Slab Geometry}

To illustrate the numerical procedure, we use only one ring (one delta function) with $c_{1}=1$ and $v_{\perp}^{1}=\sqrt{2} v_{t h i}$ to calculate $\bar{\Phi}(x)$ in the slab geometry. The 4-point averaging process is shown in Fig. 1 where the grid spacing is equal to the ion gyroradius. Here $\tilde{\boldsymbol{\Phi}}_{i, j}$ at a grid point $i, j$ is the average of $\overline{\mathbf{\Phi}}(\mathbf{R})$ on a ring (solid) which is centered at this point with a radius of $\rho=\sqrt{2} \rho_{i}$. Thus, $\tilde{\mathbf{\Phi}}_{i, j}$ is simply the 4-point average of $\langle\Phi(\mathbf{R})\rangle_{(i \pm 1, j \pm 1)}$. The value of $\bar{\Phi}(R)$ at each of these points $(i \pm 1, j \pm 1)$, in turn, is calculated by averaging $\Phi(\mathbf{x})$ on another ring (dashed) centered at that point with the same radius. This second averaging process is also done by the 4-point average. The whole process gives,

$$
\bar{\Phi}_{i, j}=\frac{1}{16}\left(4 \bar{\Phi}_{i, j}+2 \Phi_{i \pm 2, j}+2 \Phi_{i, j \pm 2}+\Phi_{i \pm 2, j \pm 2}\right),
$$


where $\Phi_{i, j}$ is the potential at grid point $i, j$.

We now put this expression of $\tilde{\Phi}$ back in Eq. 2. The resulting gyrokinetic Poisson equation is in a matrix form. It can then be solved either by an iteration scheme or by a direct matrix inversion operation. Since $\Gamma_{0}$ is always smaller than 1 and only varies in a limited range, the convergence for the iteraition is guaranteed and is actually quite fast. When the electron is adiabatic and $\tau=1$, we have found that only $3 \sim 4$ iterations are needed.

To calculate $\tilde{\boldsymbol{\Phi}}$ near the boundary, the grid array is extended beyond the simulation boundary, and the $\Phi$ 's outside the boundary are set by the appropriate $\Phi$ 's inside depending on whether it is periodic or reflective boundary condition. Only those $\tilde{\boldsymbol{\Phi}}$ 's inside or on the boundary needed to be calculated. Thus, the boundary condition is automatically implemented in this process of calculating $\tilde{\Phi}$.

In the usual FFT approach, numerical filter of the form of $f_{k}=\exp [-(\mathrm{a}$. k) ${ }^{2}$ ] is usually applied in the $\mathbf{k}$-space, $f_{k} \Phi_{k} \rightarrow \Phi_{k}$, to cutoff the high $\mathbf{k}$ mode fluctuations in order to reduce the simulation noise. Here $a$ represents the lower bound of the wavelength of interest. Similar filtering can be constructed by numerical smoothing in the configuration space. To simplify the analysis, let us consider the one-dimension geometry with a uniform grid spacing of $\Delta x$. We can cut off short wavelength modes by replacing the potential at each grid point with a weighted average at several adjacent grid points. Let us assume,

$$
\Phi(x)=\sum_{n} \alpha_{n} \delta\left(x^{\prime}-x \pm n \Delta x\right) \Phi^{\prime}\left(x^{\prime}\right)=\sum_{n} \alpha_{n} \Phi^{\prime}(x \pm n \Delta x),
$$

where $\alpha$ is the weighted factor, and $\Phi(x)$ and $\Phi^{\prime}\left(x^{\prime}\right)$ are the potential after and before the numerical smoothing, respectively. Then, from the convolution theorem, this configuration space smoothing is equivalent to applying the following form factor in $\mathbf{k}$-space,

$$
f_{k}=\sum_{n} \alpha_{n} \cos (k n \Delta x)
$$

where $\alpha_{n}$ 's are set by choosing a desired form factor of filtering. For example, simple smoothing with $\Phi_{i, j}=\frac{1}{4}\left(2 \Phi_{i, j}^{\prime}+\Phi_{i \pm 1, j}^{\prime}\right)$ gives the filtering of the form $f_{k}=\cos ^{2}(k \Delta x / 2)$, which is similar to that of $f_{k}=e^{-a^{2} k^{2}}$ with $a=\Delta x / 2$.

To test the new Poisson solver, nonlinear simulation for a simple system of $\eta_{i}$ instability has been carried out in a shearless slab. The mode history of electrostatic potential using both the FFT and the iteration scheme of the 
present solver with 4 iterations is plotted in Fig. 3 and Fig. 4, respectively. It is shown that the present scheme gives the results in excslient agreement with those of FFT method which is exact for slab geometry.

\section{B. Toroidal Geometry}

In this subsection, we compare the results of the present scheme with the exact solution of $\mathrm{Li}$ et al. ${ }^{3}$ in toroidal geometry. The potential in this case can be expressed as Bessel-Fourier series in term of the toroidal coordinates $(r, \theta, \phi)$,

$$
\Phi(\mathbf{x}) \equiv \Phi(r, \theta, \phi)=\sum_{l, m, n}^{\infty} \Phi_{1 m n} J_{m}\left(\alpha_{m} r\right) e^{i(m \theta+n \phi)},
$$

where $r, \theta, \phi$ are in the minor radius, poloidal and toroidal direction, respectively, $\alpha_{m l}$ is the $l$-th zero of $J_{m}$, and the minor radius is normalized to 1 . This set of trial functions satisfies the regularity condition at $r=0$ and the boundary condition $\Phi(r=1)=0$.

Assuming a pure toroidal magnetic field and a circular cross section, one can carry out the gyrophase averaging procedure to obtain,

$$
\overline{\boldsymbol{\Phi}}(\mathbf{R}) \equiv \overline{\boldsymbol{\Phi}}\left(r_{0}, \theta_{0}, \phi\right)=\sum_{l, m, n}^{\infty} \Phi_{l m n} J_{0}\left(\alpha_{m !} \rho_{i}\right) J_{m}\left(\alpha_{m l} r_{0}\right) e^{i\left(m \theta_{0}+n \phi\right)}
$$

where $r_{0}, \theta_{0}$ and $\phi$ are the guiding center positions in the toroidal coordinate. Again assuming a Maxwellian equilibrium, the secord gyrophase averaged potential $\tilde{\Phi}$ can be expressed as,

$$
\tilde{\Phi}(x)=\sum_{l, m, n}^{\infty} \Gamma_{0}\left(\alpha_{m l}^{2} \rho_{i}^{2}\right) \Phi_{l m n} J_{m}\left(\alpha_{m l} r\right) e^{i(m \theta+n \phi)}
$$

Utilizing this relation, the gyrokinetic Poissou equation can be expressed in the Bessel-Fourier space as a matrix equation,

$$
\frac{\tau}{\lambda_{D}^{2}} \sum_{l, m, n} \Phi_{l m n}\left[1-\Gamma_{0}\left(\alpha_{m l}^{2} \rho_{i}^{2}\right)\right] J_{m}\left(\alpha_{m l} r\right) e^{i(m \theta+n \phi)}=-4 \pi e\left(\overline{n_{i}}-n_{e}\right) .
$$

This equation is coupled in the $\theta$ and $r$ directions due to the dependence of $\rho_{i}$ on $\theta$ and $r$.

We now can compare the relative accuracy of the new Poisson solver with the FFT-approach in toroidal geometry. For this purp'sse we assume 
a uniform magnetic field and temperature profile. Thus, $\rho_{i}$ is independent. of $r, \theta$ and $\phi$, and the the gyrokinetic Poisson equation is decoupled for different $l, m, n$ modes. Given charge density as the sum of a finite siries of Bessel-Fourier trial functions, the potencial can be obtained analytically using Eq. 9. This result can then be compared with those calculated from the same charge density by the generalized Poisson solver and the FFT method.

For the new Poisson solver, the $r-\theta$ plane is divided into equal area cells (uniform grid spacing in $r^{2}-\theta$ ). The hreakdown of the number of grid points in $r$ and $\theta$ direction is arranged in such a way that each direction has the same resolution near the half minor radius where most interesting physics usually takes place. The FFT method uses the usual $x-y$ coordinate, and the same number of grid points is used for both approaches.

Fig. 5 depicts the potential as a function of $r$ calculated by analytical solution (solid line), the generalized Poisson solver (dotted line) and FFT method (dashed line), respectively. It is shown that the new Poisson solver is more accurate than the FFT method although the same number of grid points is used. The different is especially obvious near the edge $(r=a)$. In this comparison, a single toroidal mode (uniform) is used, and the corresponding perpendicular spectral of the potential, as shown in Figs. 6 and 7, are similar to those of nonlinear $\eta_{i}$ instability toroidal simulations. In the calculation, the grids spacing for FFT is $\delta x=\rho_{i}$, and he orea element for the new Poisson solver is $\delta r^{2} \delta \theta=\rho_{i}^{2}$. When more grid points are used, the results for both methods approach the exact solution.

Next we look at the modification to the potential due to the background profile variation. Assuming a temperature profile as shown in Fig. 8, the potential calculated by the generalized Poisson solver taking into account the profile effect is shown as dotted line in Fig. 9. The solid line in the same figure represents the analytical potential calculated without the background profile effect. The same charge density that produces the potential in Fig. 5 is used for the calculation. Although the different is modest, it can accumulate each time step in the simulation and may affect the steady state results.

The new Poisson solver has been implemented on both the vector machine (Cray-C90) and the massively parallel machine (CM-5 and T3D). The computational cost depends on the complexity of the geometry. In term of the CPU time, this solver is faster than that the FFT method in a shearless slab. In the realistic tokamak equilibrium, it roughly takes $2 \sqrt{\rho_{i}^{2} / \delta r^{2} \delta \theta} \mu s$ per grid point per iteration. This is comparable to the computational cost 
of the particle pushing part of the code assuming one particle per cell in the simulation.

\section{CONCLUSION}

A non-FFT gyrokinetic Poisson solver has been developed for general geometry gyrokinetic particle simulation. The new technique can also take into account the spatial dependence of equilibrium profile in the gyrokinetic Poisson equation. This approach employs local operations, and therefore, it is useful for global gyrokinetic simulation in general geometry using massively parallel computers.

\section{ACKNOWLEDGMENTS}

The authors would like to thank Dr. S. E. Parker, Dr. J. C. Cummings and Dr. J. V. W. Reynders for useful discussions. This work was supported by the U.S. Department of Energy under Contract No. DE-AC0276-CHO3073. 


\section{References}

${ }^{1}$ W. W. Lee, J. Comput. Phys. 72, 243 (1987).

${ }^{2}$ M. J. LeBrun, T. Tajima, W. Horton, and Y. Kishimoto, in Int. Sherwood Fusion Theory Conference, p. 1D14, Dallas, Texas, 1994.

3J. Li, W. W. Lee, and S. E. Parker, 1994, private communication.

${ }^{4}$ D. H. E. Dubin, J. A. Krommes, C. Oberman, and W. W. Lee, Phys. Fluids 26, 3524 (1983).

\section{FIGURE CAPTIONS}

FIG. 1. The schematic procedure for the calculation of $\tilde{\phi}$ using one delta function.

FIG. 2. Approximate $\Gamma_{0}$ calculated using three delta functions (dotted line) is compared with the exact $\Gamma_{0}$ (solid line).

FIG. 3. The mode history of the electrostatic potential using the FFT method.

FIG. 4. The mode history of the electrostatic potential using the generalized Poisson solver.

FIG. 5. Electrostatic potential at $\theta=0$ vs. minor radius based on a given charge density calculated by the analytic method (solid), the FFT method (dashed) and the generalized Poisson solver (dotted).

FIG. 6. $k_{r}$ spectrum calculated from model charge density.

FIG. 7. $k_{\theta}$ spectrum calculated from model charge density.

FIG. 8. A model for the radius temperature profile.

FIG. 9. Electrostatic potential at $\theta=0$ vs. minor radius using the model temperature profile of Fig. 9. 


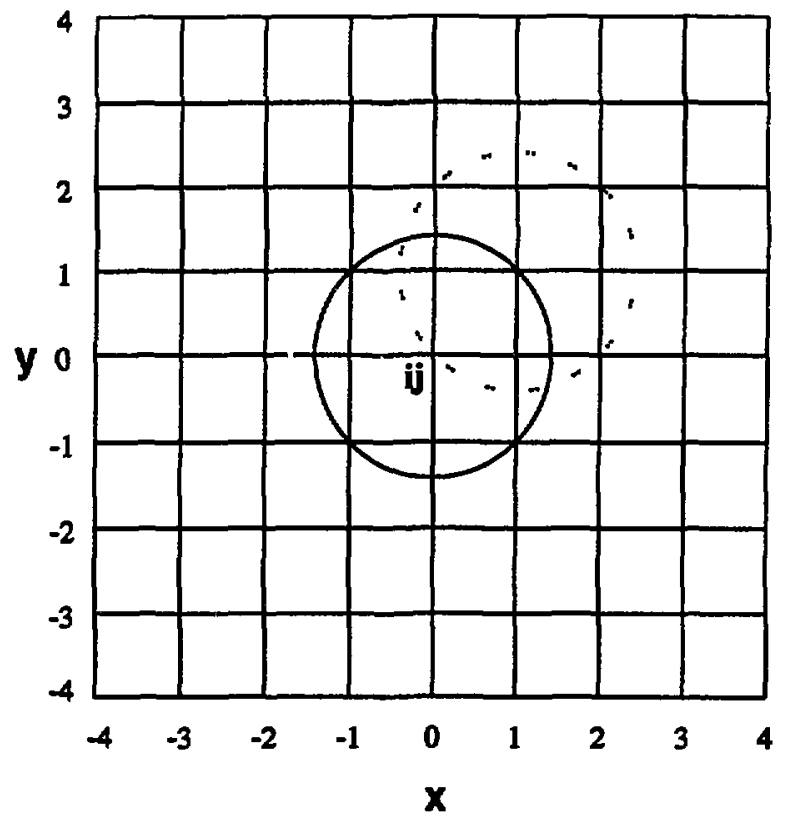

Figure 1: 


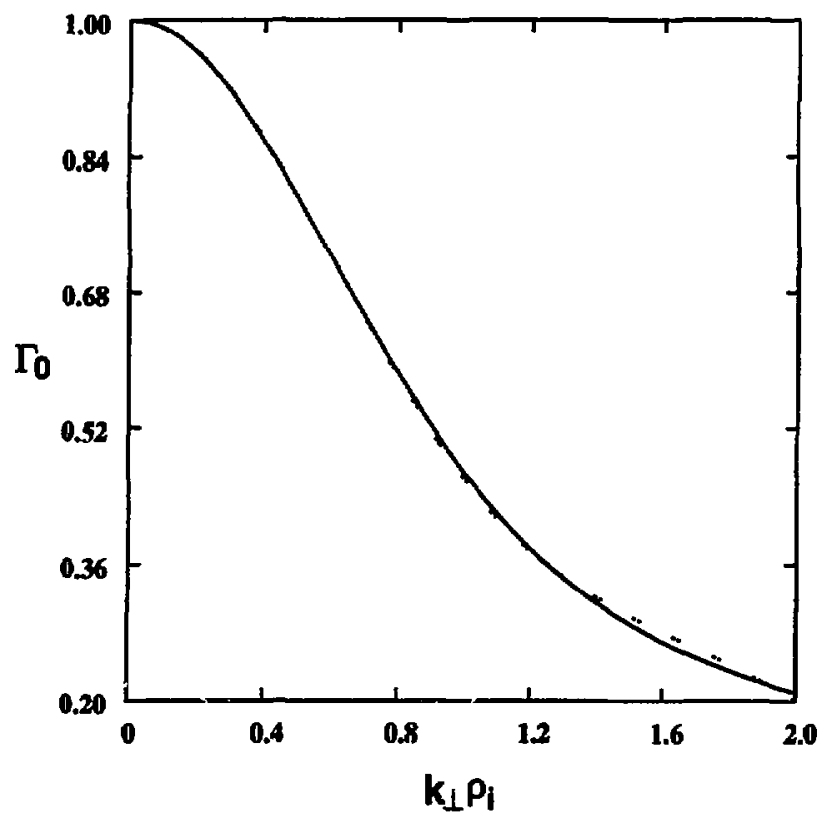

Figure 2: 


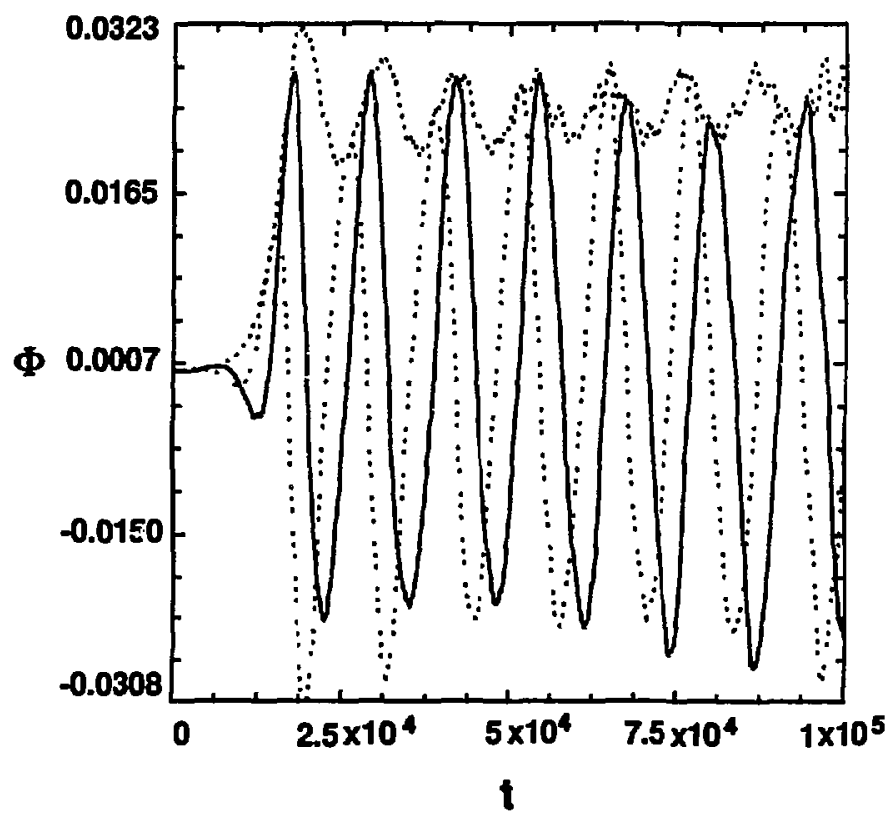

Figure 3: 


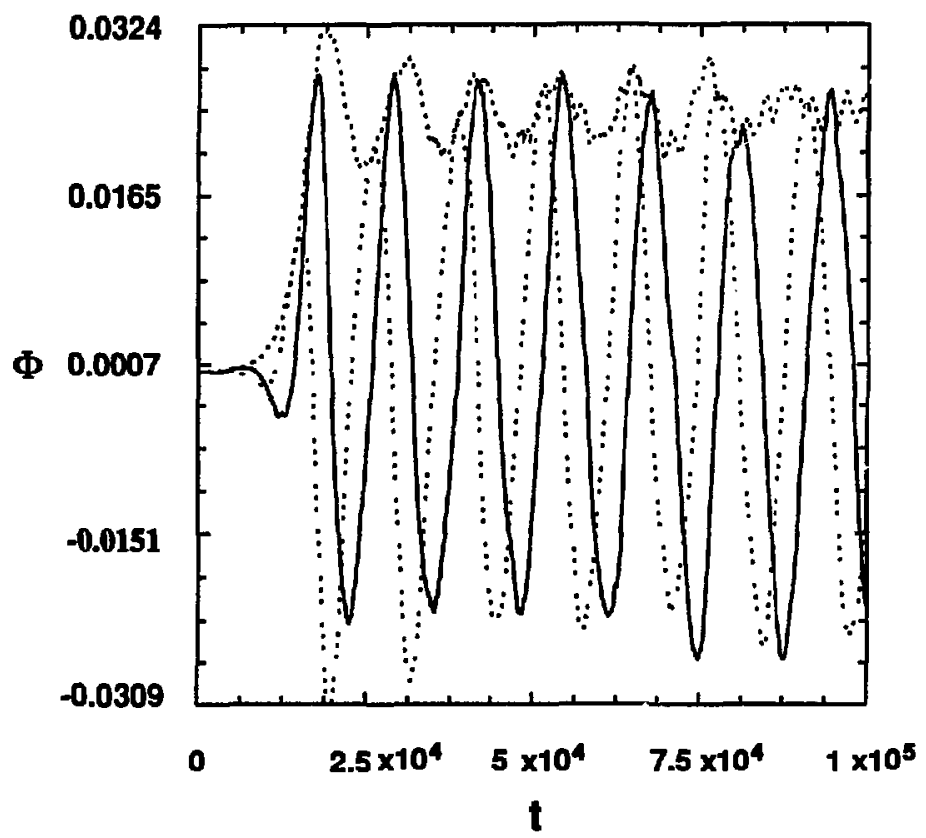

Figure 4: 


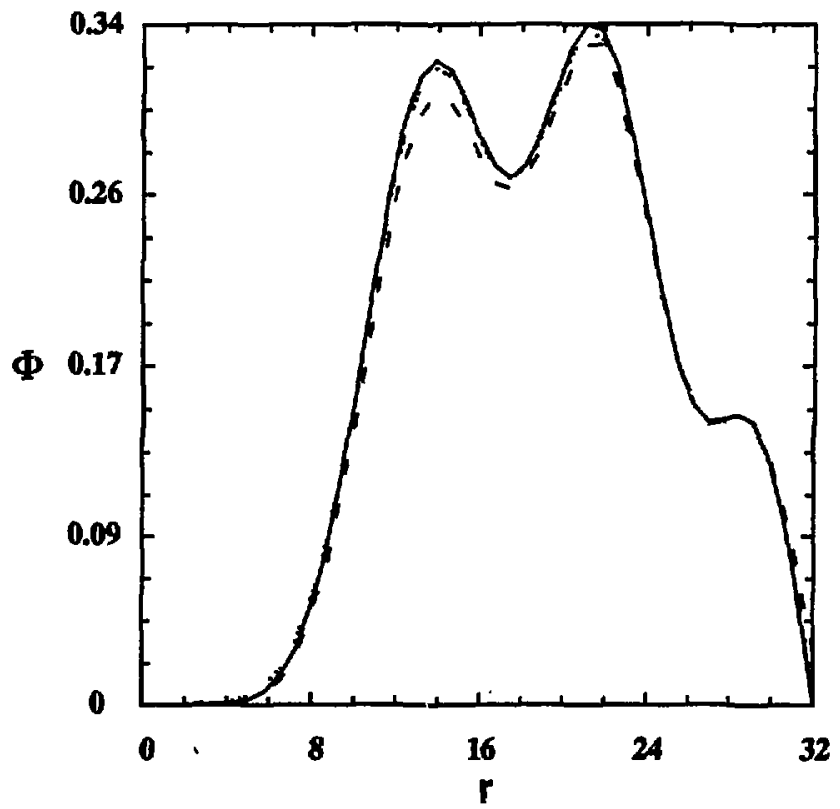

Figure 5: 


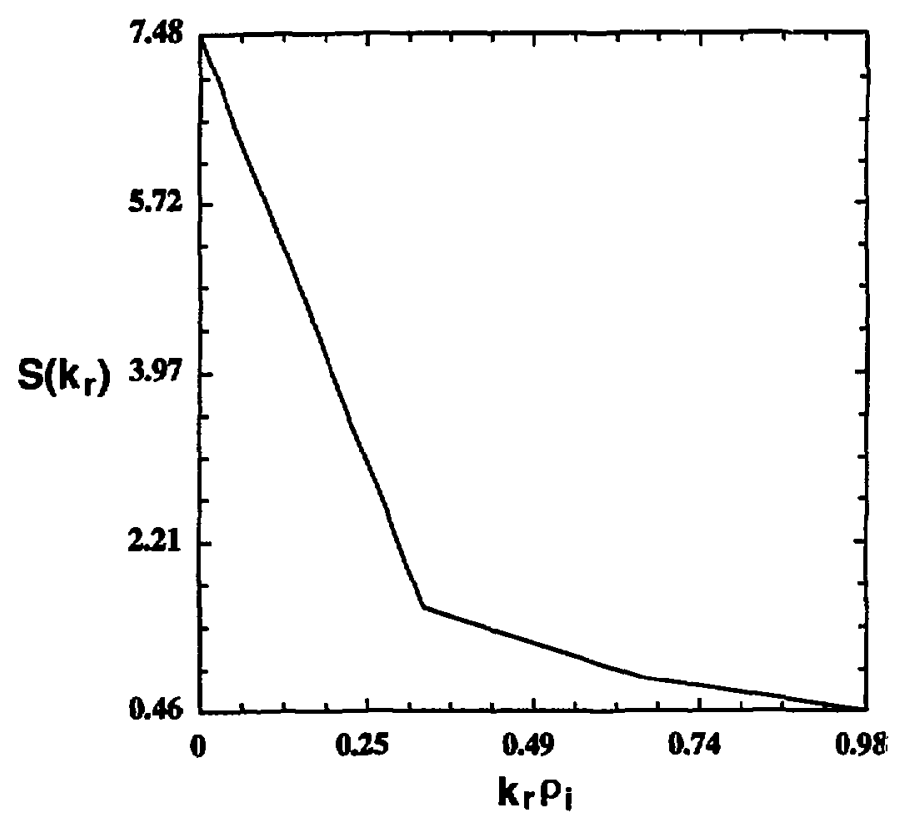

Figure 6: 


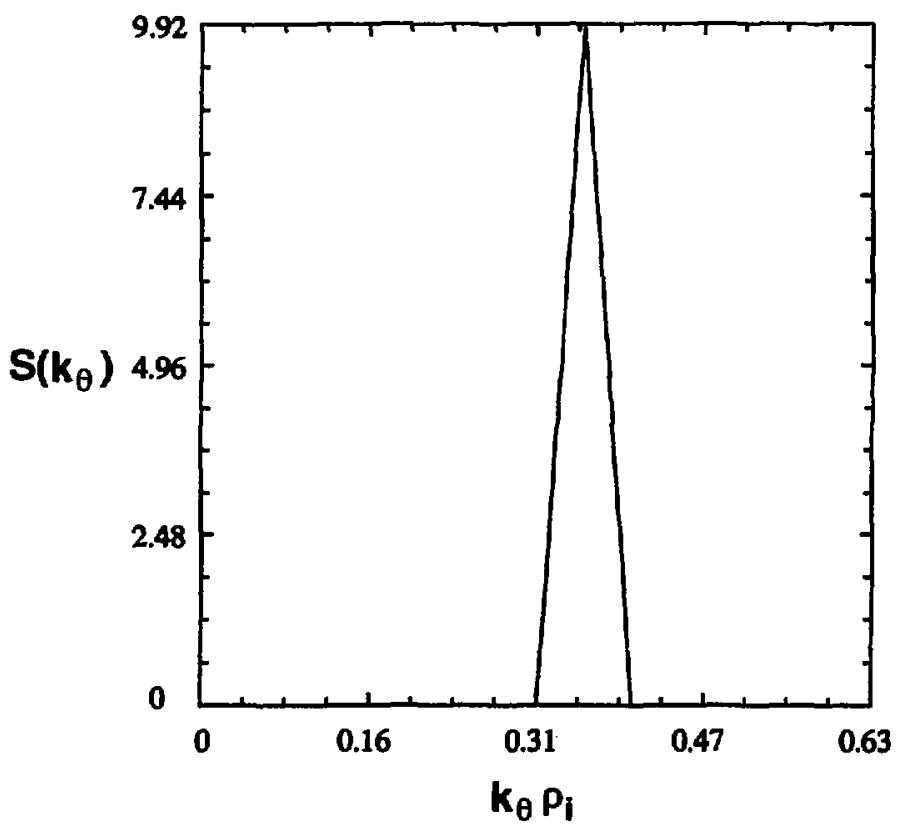

Figure 7: 


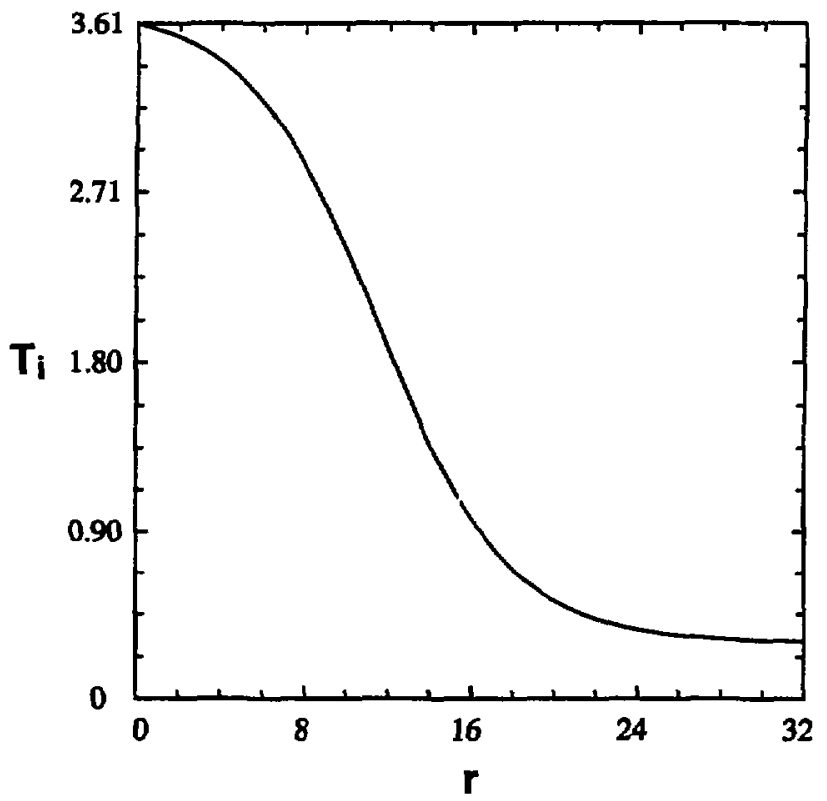

Figure 8: 


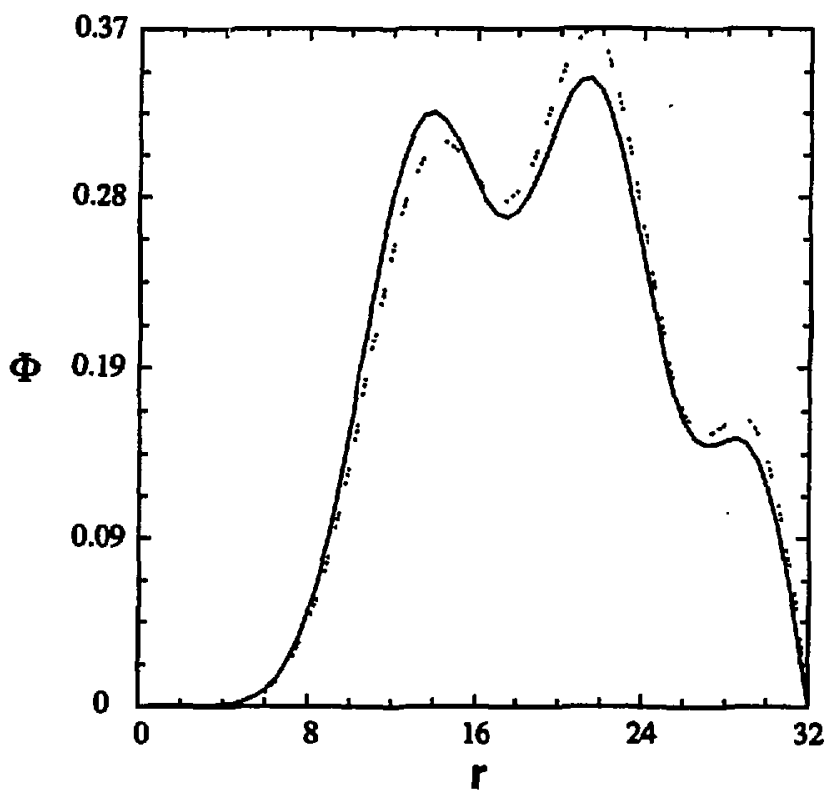

Figure 9: 
Dr. F. Peoboni, Univ. of Wollongong, AUSTRALIA

Prof. A.C. Cross, Univ. of Sydnoy, AuSTRALIA

Pleema Rosearch Lab., Austration Nat. Univ., AUSTRALKA

Prot. I.R. Jonet, Flindon Univ, AUSTRALIA

Prof. F. Cep, Inat for Theoretieal Phycies, AUSTRIA

Prof. M. Hoindier, inctiut for Thaonctache Physik, AUSTFIA

Prof. M. Gooseans, Astronomiech inetiturt, BELGUM

Ecole Royde Miltuire, Leb. do Pby. Placmars, BELGIUM

Commission-Europaen, DG. XII-Fusion Prog., BELGIUM

Prof. R. Bouciqut, Rikeuniveraitin Gent, BELGIUM

Dr. P.H. Sukanake, Inatituto Fition, BRAZIL

Prol. Dr. I.C. Nescimenso, Instivio Fivica, Seo Paub, BRAZIL hatituto Heciond Do Pesquieas Espacisie-INPE, BPUZIL Documents Orice, Alomic Energy of Cenada L4., CANADA Ma. M. Morin, CCFMT Tokamak do Varennes, CANAOA

Dr. M.P. Bechynakj, MPB Technologies, Inc., CANADA

Dr. H.M. Skersgand, Univ. of Sakkachewen, CANADA

Prol. J. Tuichmann, Univ. of Montred, CaNADA

Prot. S.P. Sreenivaem, Univ. of Celgury. CANADA

Prot. T.W. Johneton, INAS-Enemio, CANAOA

Dr. R. Bowon, Centse canadion de fusion megndique, CANADA

Dr. C.P. James., Univ. of Aberth, CANADA

D. P. Lutice, Konensketho Unimertzita, CZECHOSLOVAKIA

The Librarian, Culham Laboralony, ENGLAND

Library, R61, Ruthertord Applesen Laboratony. ENGLAND

Mrs. SA. Hutchinson, JET Lbrary. ENGLAND

Dr. S.C. Shama, Univ. of South Pecilic, FWI ISLANDS

P. Mutonen, Univ. of Helsinkj, FINLAND

Prot. M.N. Butetce, Ecola Pohyechnique, FRANCE

C. Mouttol, Lab. de Physique des Mitioux lonisfs, FRANCE

J. Padat, Cenvcadarache - Bat 506, FRANCE

Prot. E. Economou, Univ. of Crew, GREECE

Mis. C. Rinni, Univ. of lomnina, CREECE

Preprint Librery, Hungarien Academy of Sci., HUNCARY

Dr. B. Dascupta, Saha hat. of Nueber Ptyrics, INDIA

Dr. P. Kew, Inst. for Plasmu Anesearch, INDIA

Dr. P. Roseney, lared inst of Technolooy, ISRAEL

Librevien, Inimmationd Conwer for Theo Phycics, ITALY

Mies C. De Peb, Associazions EURATOMENEA, ITALY

Dr. G. Groseo, Istuto of Ficica ded Plesma, ITALY

Prof. G. Rostenoni, latitus Cas lonizzeti Del Car, ITALY
Dr. H. Yamalo, Toshiba Res a Devel Cenbr, JAPAN

Prol. I. Kawakami, Hiroshima Univ., JAPAN

Prol. K. Nithikenwa, Hiroshima Univ., JAPAN

Librarion, Naka Fusion Rotearch Establishmont, JAERI, JAPAN

Diretor, depen Alonic Enorgy Rosaurch lost, MAPN

Prof. S. Itoh, Kywehe Univ., JAPAN

Reseawch Inb. Cr., Nationed Instit for Fusion Scienco, JAPAN

Prof. S. Tenake, Kyoto Univ., JAPAN

Lbrery, Kyolo Univ., JAPAN

Prof. N. Inous, Univ. of Tokyo, LAPAN

Secretany, Plamm Saction, Electrolechnicel Leb., JAPAN

D. O. Murai, Kumamow Inat. of Technology, JAPAN

Dr. G.S. Lea, Korea Bacie Sci. Cr., KOPEA

J. Hyeon-Sook, Korea Atomio Enory Raceerch Inst., KOREA

D.I. Choi, The Kores Adv. Inet of Sa. 1 Tech., KOAEA

Prof. B.S. Liloy, Univ. of Wrikab, NEW ZEALAND

Inst of Phycics, Chinece Aad Sai PEOPLE'S REP. Of CHINA

Librey, Inst of Plasma Phycics, PEOPLE'S REP. Of CHINA

Tsinghua Univ. Librery, PEOPLE'S REPUBLIC OF CHINA

Z. Li, S.W. Ind Phyaics, PEOPLE'S REPUBUC OF CHINA

Prof. J.AC. Cubrd, Instivto Superior Tecnico, PORTUGAL

Prof. M.A. Hembery, Univ. of Natal, S. AfFica

Prof. D.E. Km, Pohang Inst. of Sci. \& Tech., SO. KOREA

Prof. C.I.E.MA.T, Fusion Division Library, SPAIN

Dr. L Sienflo, Univ. of UMEA, SWEDEN

Library. Royel hat. of Technology, SWEDEN

Prof. H. Whatmeon, Chainers Uhiv, of Tech., SWEDEN

Centre Phys. Dos Pinemas, Ecole Pohtuch, swizerLAND

Bibliotheck, Inct. Voor Plasma-Fysica, THE NETHERLANDS

Ast. Prol. Dr. S. Cesir, Middle East Tech. Univ., TUFKEY

Dr. V.A. Clukhikh,Sci. Res. Inst. Eloctrophys. I Apperatus, USSR

Dr. D.D. Ayrubv, Siberian Brench of Academy of Sa., USSR

Dr. G.A. Elienev, I.V. Kurchalov Inat, USSR

Librein, The Ukr.SSR Academy of Sciences, USSA

Dr. LM. Kovizhnykh, ins. of Genord Phycics, USSA

Kemforechungcaniege GmbH, Zentrabibiothek, W. CEPMANY

Bibliothek, Inst. For Plasmaforechung, W. GEAMANY

Prof. K. Sahinder, Puhr-Univercitit Bochum, W. GEPMANY

Dr. F. Whoner, (ASDEX), Mux-Planck-hatitut, W. GERMANY

Librarien, Max-Ptenck-Institut, W. GERMANY 